\title{
Bleeding Meckel's diverticulum diagnosis: an unusual indication for computed tomography
}

\author{
D. Danzer, ${ }^{1}$ P. Gervaz, ${ }^{1}$ A. Platon, ${ }^{2}$ P.-A. Poletti ${ }^{2}$ \\ ${ }^{1}$ Clinic of Digestive Surgery, Hôpital Cantonal Universitaire de Genève, 24 rue Micheli-du-Crest, 1211 Geneva, Switzerland \\ ${ }^{2}$ Departement of Radiology, Hôpital Cantonal Universitaire de Genève, 24 rue Micheli-du-Crest, 1211 Geneva, Switzerland
}

\begin{abstract}
Despite the wide use of modern investigation techniques, the diagnosis of complications related to Meckel's diverticulum (MD) remains difficult. Arteriography is commonly indicated for acute bleeding, and radionuclide scans may help in identifying the site of intestinal hemorrhage. In contrast, computed tomography (CT) is usually considered little use in the diagnosis of bleeding MD. We present the case of a young patient with massive gastrointestinal hemorrhage, in whom the diagnosis of MD bleeding was preoperatively made with contrastenhanced CT after two negatives arteriographies.
\end{abstract}

Key words: Meckel's diverticulum-Bleeding-Computed tomography_Arteriography_-Imaging.

The total lifetime complication rate from Meckel's diverticulum (MD) is $4 \%$ [1]. Bleeding occurs as a result of ileal mucosal ulceration adjacent to acid-producing heterotopic gastric mucosa [2]. Despite the wide use of modern imaging techniques, the diagnosis of bleeding MD remains difficult; in some series, fewer than $10 \%$ of symptomatic MDs are correctly diagnosed preoperatively [3].

Enteroclysis is considered the examination of choice for MD diagnosis; angiography and radionuclide scans are indicated in cases of acute bleeding and have proven useful in identifying the source of hemorrhage, because the distinction between a diverticulum and an intestinal loop is usually impossible on computed tomography (CT) [4]. We report the case of a young patient presenting with gastrointestinal bleeding. Contrast-enhanced CT demonstrated extravasation of contrast in an MD, whereas two

Correspondence to: P. Gervaz

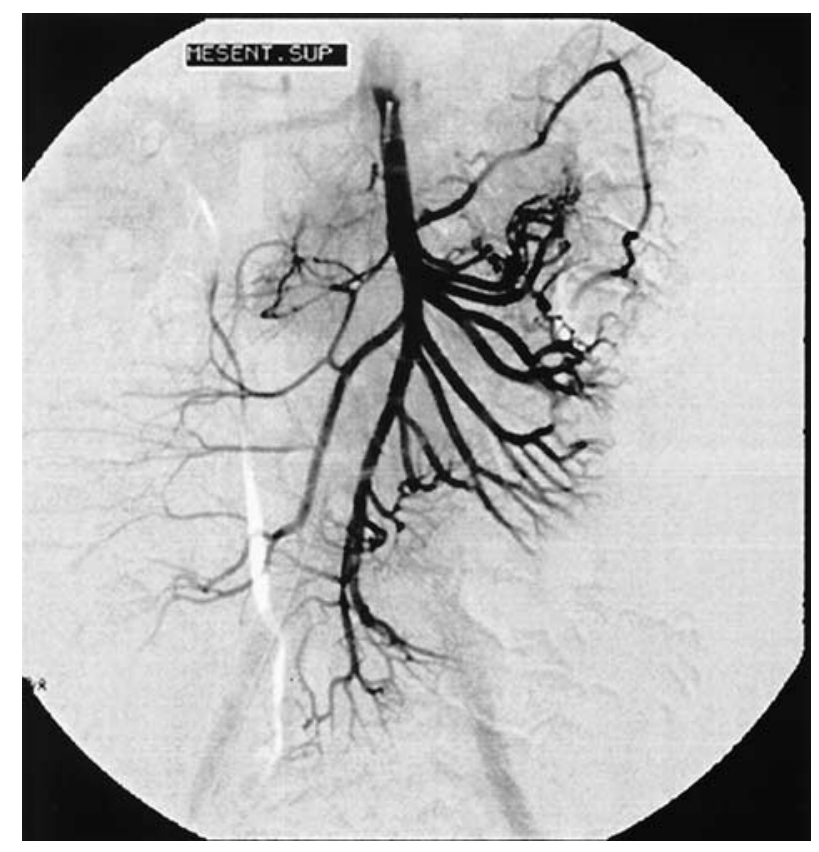

Fig. 1. Superior mesenteric arteriography shows no sign of contrast extravasation.

successive angiographies had previously failed to identify the site of hemorrhage.

\section{Case report}

A 22-year-old male was admitted to our intensive care unit after a first episode of melena associated with hematochezia. In the emergency room, the patient had massive blood loss and presented with orthostatic hypotension. The patient was in moderate distress and pale; blood pressure was $90 / 30 \mathrm{mmHg}$ and the pulse was 76 beats/ $\mathrm{min}$. The abdominal examination was negative, except for 

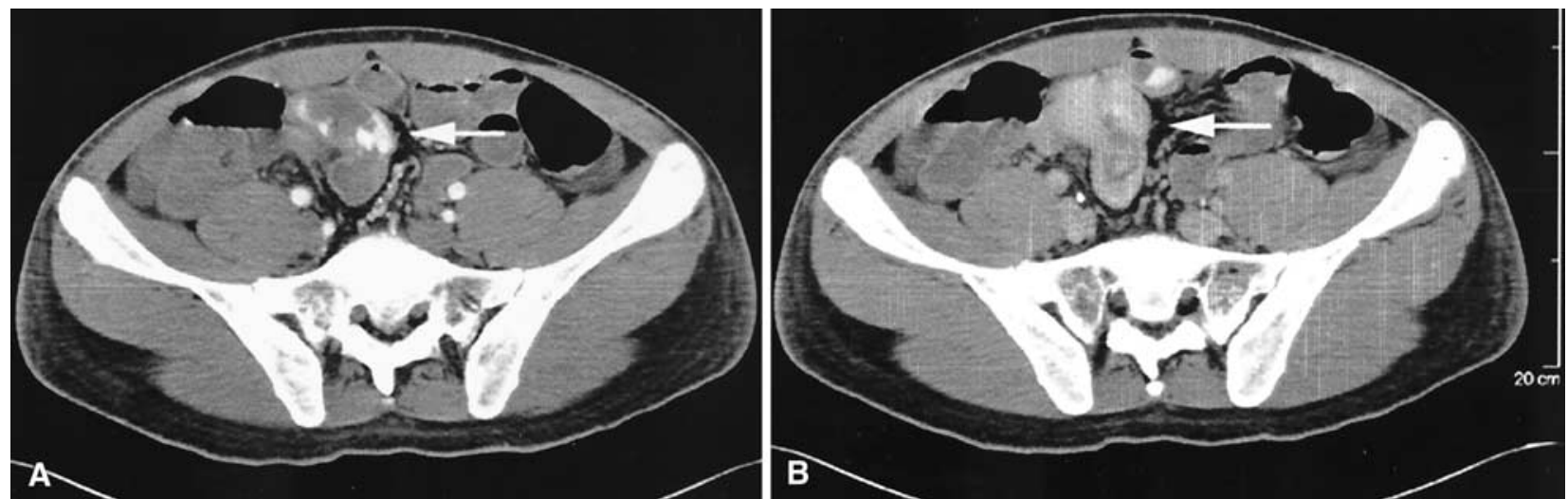

Fig. 2. A Contrast-enhanced CT, arterial phase. Active bleeding at the collar of a blind digestive structure is seen arising from the distal ileum (arrow). B Contrast-enhanced CT, late phase. Contrast accumulation in the blind loop connected to the terminal ileum (arrow) demonstrates hemorrhage in MD.

increased bowel sounds. Initial hematocrit was measured at $22 \%$ (hemoglobin, $73 \mathrm{~g} / \mathrm{L}$ ). The patient was resuscitated with perfusion of intravenous crystalloid, and he received $4 \mathrm{U}$ of blood, with subsequent stabilization of hemodynamic parameters. An upper gastrointestinal endoscopy was normal, and colonoscopy demonstrated bleeding proximal to the ileocecal valve.

During the second day of hospitalization, the patient was stable but had continuing evidence of intestinal bleeding; an initial arteriography of the superior and inferior mesenteric arteries, with selective catheterization of the ileocolic artery, demonstrated no contrast extravasation or persistence of a right vitelline artery. Overnight, the patient had a third episode of blood loss, which motivated a second angiography to localize the bleeding source before planning any surgery. Arteriography did not show the source of the hemorrhage (Fig. 1).

After a third bleeding episode, contrast-enhanced CT, arterial phase, showed active bleeding at the collar of a blind digestive structure arising from the distal ileum (Fig. 2A), the venous and late phases of the examination confirmed the contrast accumulation in this blind loop (Fig. 2B), indicating hemorrhage in an MD. The patient was taken to the operating room, the preoperative diagnosis of bleeding MD was confirmed, and an ileal resection was performed. Pathologic examination of the specimen showed a true diverticulum $60 \mathrm{~cm}$ from the ileocecal valve, $25 \mathrm{~mm}$ in length and $15 \mathrm{~mm}$ in diameter, at the antimesenteric border of the ileum, with heterotopic gastric mucosa (Fig. 3). The patient was discharged 6 days after the procedure without complications.

\section{Discussion}

Bleeding is a common complication of MD and in most cases is associated with the presence of gastric hetero-

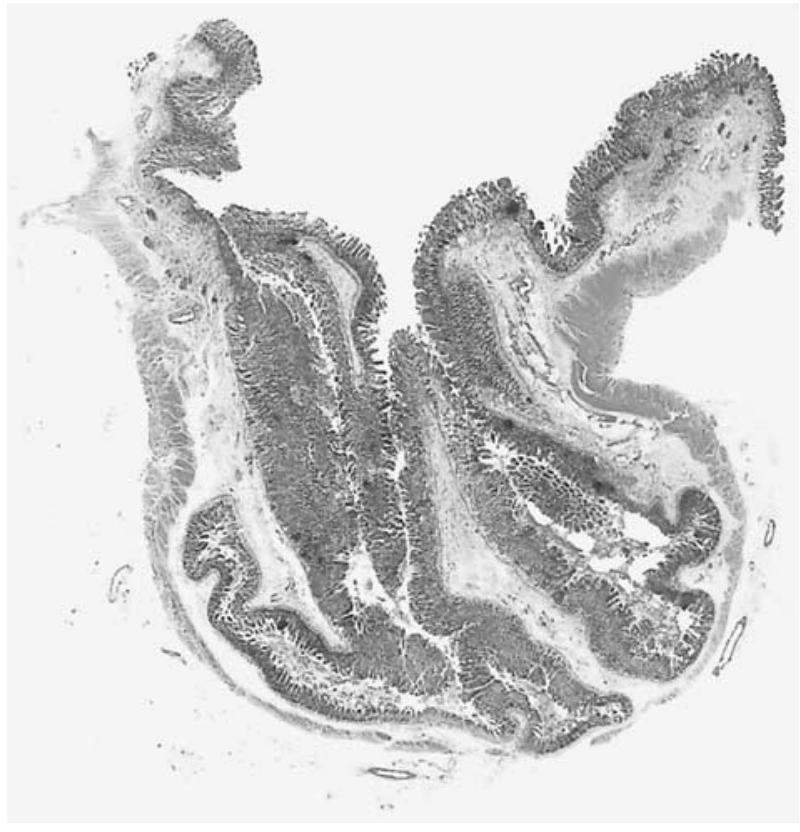

Fig. 3. Overview of the resection tissue. Inside the MD lumen, the wider mucosa folds up. The intestinal muscular wall gets thinner along the MD. Hematoxylin and eosin stain; original magnification, $1 \times$.

topic mucosa. Preoperative diagnosis of complicated MD remains a challenge $[5,6]$, and preoperative diagnosis of bleeding MD with contrast-enhanced CT, as described in this report, is exceptional for two reasons. First, the MD must be large enough to be detected and differentiated from surroundings bowel loops. In our case, the MD was large with a wide implantation. Second, and most importantly, the bleeding must be active before the contrast is excreted by the kidneys.

Apart from enteroclysis, the technique of choice for MD imaging is radionuclide scan with scintigraphy using 
99mTc-pertechnetate or $99 \mathrm{mTc}$-labeled red blood cells. Both techniques have high sensitivity, but their performance can be decreased in case of active bleeding, which induces a washout of the radioisotope in the MD [6]. Red blood cell-labeled scintigraphy can detect active bleeding of at least $0.1 \mathrm{~mL} / \mathrm{min}$, but this technique is time consuming. Mesenteric arteriography is very specific during active bleeding and may demonstrate an accumulation of contrast in a saccular lesion or a right vitelline artery. Unfortunately, the sensitivity is low because active bleeding cannot be detected under $0.5 \mathrm{~mL} / \mathrm{min}$ [7].

The utility of CT in the diagnosis of MD is, according to many investigators, anecdotal $[1,4,8]$. Portnoy et al. [9] reported a case of MD diagnosed by CT; the characteristic feature was a well-demarcated saccular lesion filled with air, fluid, or oral contrast, attached to a distal small bowel loop, and surrounded by infiltrated fat. Miele et al. [10] reported four cases of obstruction due to a MD diagnosed preoperatively. In the present case, CT identified not only the blind digestive loop connected to the distal ileum but also the active bleeding within the diverticulum. This current case showed that CT after negative angiography can be useful when the diagnosis of a bleeding MD is suspected.

\section{References}

1. Leijonmarck CE, Bonman-Sandelin K, Frisell J, et al. Meckel's diverticulum in the adult. Br J Surg 1986;73:146-149

2. Kasumoto H, Yoshida M, Takahashi I, et al. Complications and diagnosis of Meckel's diverticulum in 776 patients. Am J Surg 1992;164:382-383

3. Yamaguchi M, Takeuchi S, Awazu S. Meckel's diverticulum: investigation of 600 patients in the Japanese literature. Am J Surg 1978;136:247-249

4. Rossi P, Gourtsoyiannis N, Bezzi M, et al. Meckel's diverticulum: imaging diagnosis. AJR 1996;166:567-573

5. Chiu EJ, Shyr YM, Su CH, et al. Diverticular disease of the small bowel. Hepatogastroenterology 2000;47:181-184

6. Groebli Y, Bertin D, Morel P. Meckel's diverticulum in adults: retrospective analysis of 119 cases and historical review. Eur J Surg 2001; 167:518-524

7. Dixon PM, Nolan DJ. The diagnosis of Meckel's diverticulum: a continuing challenge. Clin Radiol 1987;38:615-619

8. Pinero A, Martinez-Barba E, Canteras M, et al. Surgical Management and complications of Meckel's diverticulum in 90 patients. Eur J Surg 2002;168:8-12

9. Portnoy O. Gayer G, Onaca N, et al. Abdominal computerized tomography in the diagnosis of Meckel's diverticulitis in the adult. IMA J Math Appl Med Biol 2001;3:982-983

10. Miele V, De Cicco ML Andreoli C, et al. US and CT findings in complicated Meckel diverticulum. Radiol Med 2001;101:230234 